\title{
Primary posterior stabilized total knee arthroplasty: analysis of different instrumentation
}

\author{
Pier Francesco Indelli ${ }^{1,2,3,5^{*}}$, Massimiliano Marcucci ${ }^{1}$, Angelo Graceffa ${ }^{3}$, Sophie Charlton ${ }^{4}$ and Leonardo Latella
}

\begin{abstract}
Background: Intercondylar femoral bone removal during posterior stabilized (PS) total knee arthroplasty (TKA) makes many cruciate substituting implant designs less appealing than cruciate retaining implants. Bone stock conservation is considered fundamental in the prevision of future revision surgeries. The purpose of this study was to compare the quantity of intercondylar bone removable during PS housing preparation using three contemporary PS TKA instrumentations.

Method: We compared different box cutting jigs which were utilized for the PS housing of three popular PS knee prostheses. The bone removal area from every PS box cutting jig was three-dimensionally measured.

Results: Independently from the implant size, the cutting jig for a specific PS TKA always resected significantly less bone than the others: this difference was statistically significant, especially for small- to medium-sized total knee femoral components.

Conclusion: This study does not establish a clinical relevance of removing more or less bone at primary TKA, but suggests that if a PS design is indicated, it is preferable to select a model which possibly resects less distal femoral bone.
\end{abstract}

Keywords: TKA, Total knee arthroplasty, Posterior stabilized, Cruciate substituting total knee arthroplasty

\section{Introduction}

The use of posterior stabilized (PS) implants in total knee arthroplasty (TKA) is becoming increasingly popular. Many designs, which are modifications of the original Insall-Burstein prosthesis (Zimmer, Warsaw, IN, USA) [1], have inherited the classical cam and post mechanism to guide anteroposterior knee motion in lieu of the posterior cruciate ligament. Potential advantages of PS TKA include the possibility of easier balancing of severe coronal and sagittal deformities (i.e., varus/valgus or recurvatum), better controlled flexion kinematics, less polyethylene sliding wear, greater weight-bearing maximal flexion, and greater posterior femoral rollback than cruciate retaining (CR) high-flexion TKA [2]. A decrement in patello-femoral contact pressure in PS TKA designs when compared to $\mathrm{CR}$ designs is another potential advantage

\footnotetext{
* Correspondence: pindelli@stanford.edu

'Centro Eccellenza Sostituzioni Articolari Toscana (CESAT), Clinica Ortopedica Universita' di Firenze, Florence 50134, Italy

2Fondazione Onlus "In Cammino", Piazza Lavagnini 1, Fucecchio, Florence 50054, Italy

Full list of author information is available at the end of the article
}

[3]. There are several potential disadvantages in the use of PS designs with respect to other CR implants, including tibial post wear and breakage [4], increased incidence of anterior knee pain [5], and implant instability especially during the midflexion phase [6].

One of the major disadvantages of PS designs is also linked to the amount of bone removed during femoral 'box osteotomy' for the PS mechanism housing compared to posterior cruciate retaining implants. Theoretically, a major preservation of bone stock would be useful in the case of future revision of the implant: in fact, the amount and location of bone loss determine which reconstructive method (cones, methaphyseal sleeves, and/or stems) will be necessary to achieve implant stability [7]. In a recent review, Huten defined previous bone resections as the first cause of bone loss in revision total knee arthroplasty [8].

The current study was performed to examine the maximum volumetric bone resection area required for the PS housing of three worldwide highly utilized posterior stabilized TKA designs: Sigma PS (De Puy, Johnson \& Johnson, Warsaw, IN, USA), Persona (Zimmer, Warsaw, IN, USA) 
and Vanguard (Biomet Orthopedics Inc., Warsaw, IN, USA). The authors compared the manufacturer's instruments (PS box cutting jigs) in order to highlight their design differences.

\section{Material and method}

The authors acquired the complete surgical instrumental set of three different PS TKA devices Sigma PS (De Puy, Johnson \& Johnson, Warsaw, IN, USA), Persona (Zimmer, Warsaw, IN, USA), and Vanguard (Biomet Inc, Warsaw, IN, USA): analysis of very small ('micro') and very large ('macro') size implants were excluded for this study. The Sigma PS TKA surgical instrumentation included two cutting jigs for small femoral sizes (sizes 2 and 2.5 ), two jigs for medium femoral sizes (sizes 3 and 4), and one jig for large sizes (size 5). The Vanguard TKA surgical instrumentation included three cutting jigs for small femoral sizes (sizes 55, 57.5, 60), three jigs for medium femoral sizes (sizes 62.5, 65, 67.5), and three jigs for large sizes (sizes 70, 72.5, 75). The Persona TKA surgical instrumentation included one cutting jig for small femoral sizes (sizes 3 to 5), one jig for medium femoral sizes (sizes 6 to 9), and one jig for large sizes (sizes 10 to 12). In an in vivo situation, the surgical step necessary for femoral box osteotomy requires application of a cutting jig to the distal part of the femur (Figure 1).

An in vitro tridimensional evaluation (anteroposterior length, mediolateral length, and thickness) of the maximum bone removal area was performed for each of the three groups of cutting jigs (small sizes, medium sizes, large sizes) through direct measurement with a millimeter

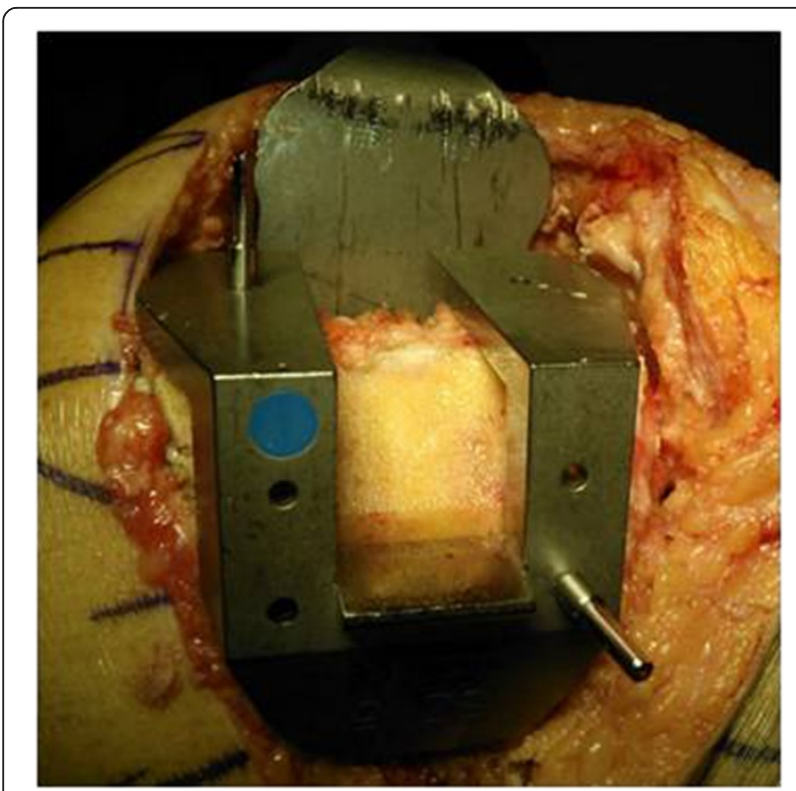

Figure 1 Right knee. Intraoperative image of a femoral jig before 'box osteotomy.' caliber (Figures 2, 3, and 4). Data was collected on an Excel spreadsheet as an average in the small, medium, and large size groups. The size of the saw blade $(1 \mathrm{~mm})$ was considered in the measurement process, as shown in Figures 2, 3, and 4.

The goal of each measurement was to evaluate the tridimensional box size of each femoral jig, not the amount of bone actually removed during the surgical procedure. Statistical analysis was performed using analysis of variance (ANOVA) test with box volume as the dependent variable.

\section{Results}

For all implant sizes (Figure 5), the Zimmer Persona jig showed a significantly inferior tridimensional box area than the Biomet Vanguard and Sigma PS $(P<0.003)$. The difference between the Zimmer Persona and the Sigma PS was even more statistically significant in small and medium size implants $(P<0.008)$.

For the small size implant, Biomet Vanguard average tridimensional box area was $11.04 \mathrm{~cm}^{3}$ compared to $24.03 \mathrm{~cm}^{3}$ for Sigma PS and to $6.18 \mathrm{~cm}^{3}$ for Zimmer Persona $(P<0.002)$.

For the medium size implant, Biomet Vanguard average tridimensional box area was $13.59 \mathrm{~cm}^{3}$ compared to $28.91 \mathrm{~cm}^{3}$ for Sigma PS and $7.69 \mathrm{~cm}^{3}$ for Zimmer Persona $(P<0.003)$.

For the large size implant, Biomet Vanguard average tridimensional box area was $16.05 \mathrm{~cm}^{3}$ compared to $36.66 \mathrm{~cm}^{3}$ for Sigma PS and $13.32 \mathrm{~cm}^{3}$ for Zimmer Persona $(P<0.006)$.

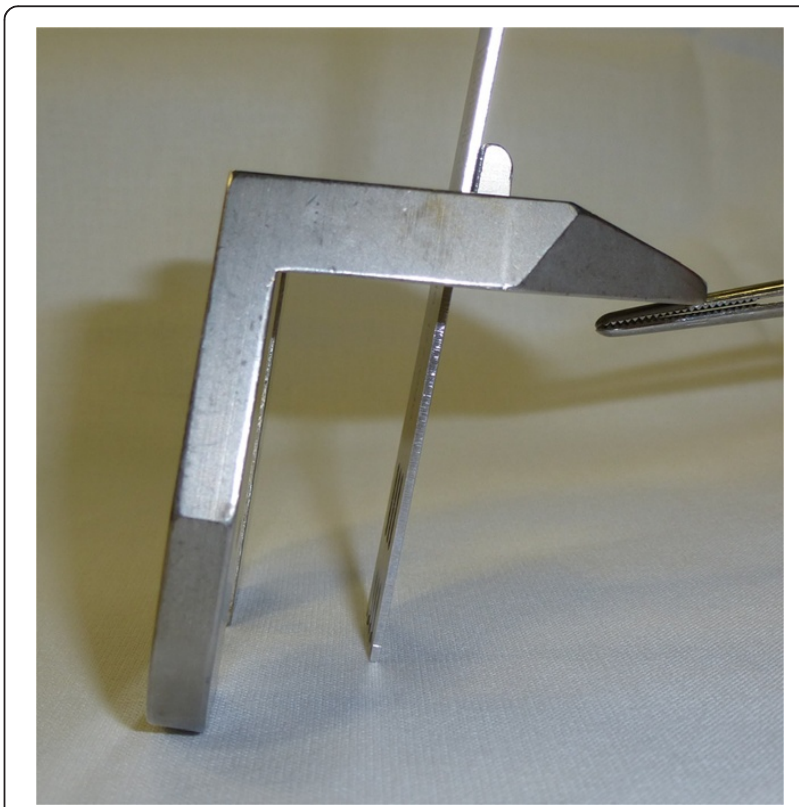

Figure 2 The De Puy Sigma PS femoral cutting jig (large sizes) is shown. An osteotome defines the bone removable area. 

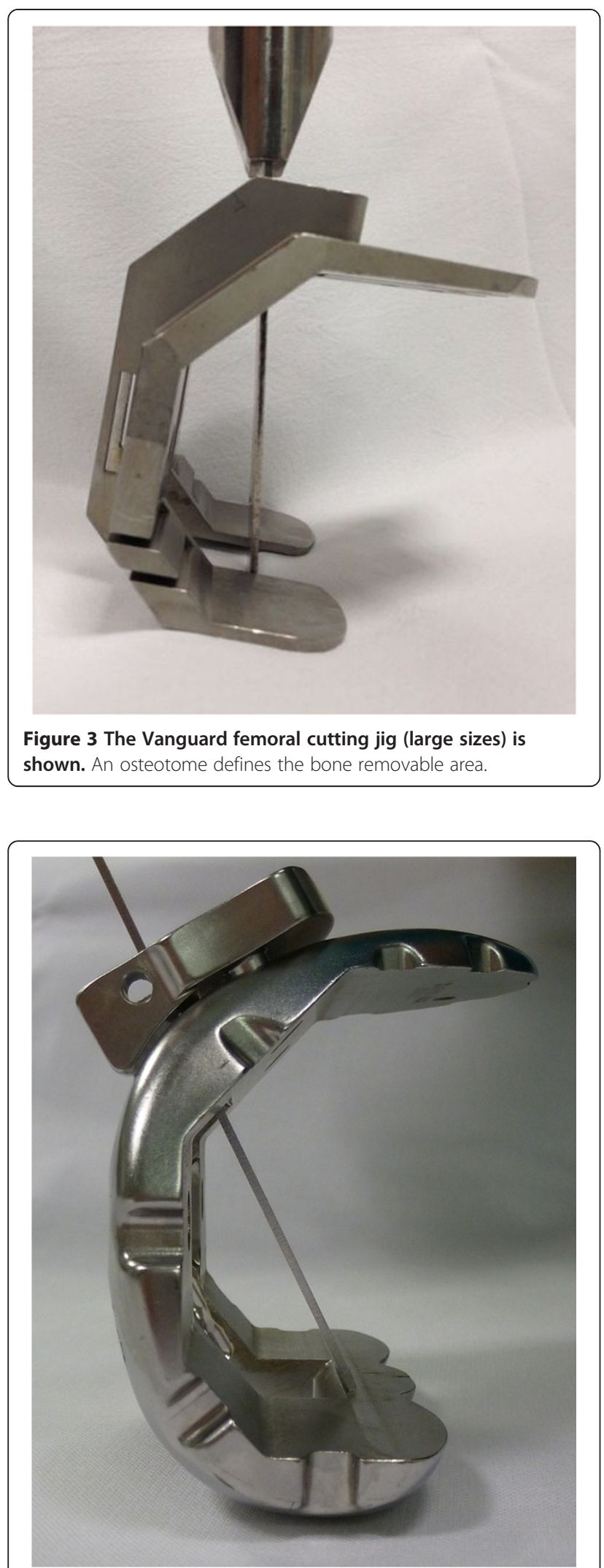

Figure 4 The Persona femoral cutting jig (large sizes) is shown. An osteotome defines the bone removable area.
The difference between the Biomet Vanguard and the Sigma PS was statistically significant $(P<0.01)$, while the difference between the Biomet Vanguard and the Zimmer Persona was not $(P>0.1)$. The difference between the Sigma PS and the Zimmer Persona was statistically significant $(P<0.008)$.

The volumetric bone resection for the PS housing does not include resection volume for the femoral lugs, typical of the Zimmer Persona design. In fact, an additional $0.59 \mathrm{~cm}^{3}$ of bone was calculated from each of the two lugs of this PS TKA design.

\section{Discussion}

Preservation or substitution of the posterior cruciate ligament in primary TKA is still a controversial issue [9]. Gait analysis [10] and in vivo [11] and in vitro [12] studies showed reproduction of a close to normal knee kinematic using either solution. A clinical comparative study between different types of TKA (cruciate retaining or posterior stabilized) with identical femoral geometry showed similar midterm outcomes with regards to the range of motion, functional outcomes, and survival rate [13].

The purpose of this study was to compare the maximum quantity of intercondylar bone removable in three highly used contemporary PS TKA designs. The implants tested in the current study have the characteristic of producing similar kinematics in the PS mechanism, including flexion angle for cam and post interaction.

The PFC Sigma (DePuy Orthopaedics Inc., Warsaw, IN, USA) TKA was introduced in 1996 as an improvement of the Press-Fit Condylar (PFC) implant (Johnson \& Johnson, Raynham, MA, USA). Design features regarding the PS housing included an updated femoral coronal geometry and a deeper and more prolonged trochlear groove to improve patellar tracking. Recently, few studies have investigated the functional outcome of the PFC and PFC Sigma knee systems [14], showing satisfactory midterm results. Few authors reported minor issues on extensor mechanism complications following the use of this implant [15]. Because of these patello-femoral issues, the PFC Sigma femoral component was redesigned, becoming available in 2009 under the name PFC Sigma PS (DePuy Orthopaedics Inc., Warsaw, IN, USA). The new principal modifications regarding the PS housing design included a 'J curve' femoral design, a new femoral box, and smoother trochlear groove edges: these design changes provided a better patellar route during ROM [5]. The Sigma PS implant shows an in vivo posterior cam-post engagement at an average of $93^{\circ}$ [16]. A new specific TKA instrumentation (high performance, HP; DePuy Orthopaedics Inc., Warsaw, IN, USA) was introduced as well, including a new PS box cutting jig which was evaluated in the current study (Figure 2). 


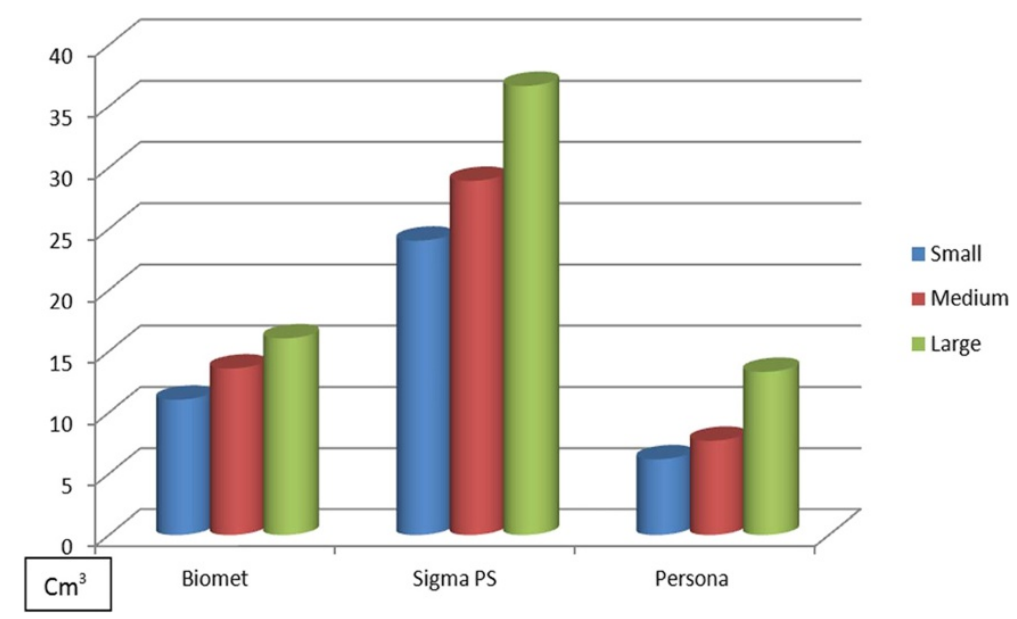

Figure 5 Bar diagram showing the maximum volumetric bone resection $\left(\mathrm{cm}^{3}\right)$ for each PS cutting jigs of the tested designs.

The Biomet Vanguard PS TKA (Biomet Inc, Warsaw, IN, USA) was first introduced in 2003. The femoral component has a rounded sagittal profile and a wide, deep, and long trochlear groove. The standard box resection cut is parallel to the distal femoral resection (Figure 3). This implant rolls and slides anteriorly until engaging the post and cam mechanism at $78^{\circ}$ of flexion. Midterm clinical results of this design are very promising too [17].

The Zimmer Persona (Zimmer, Warsaw, IN, USA) design was born following the experience of the InsallBurstein PS knee, which had a cam and post articulation at $60^{\circ}$ of knee flexion and the NexGen Legacy (Zimmer, Warsaw, IN, USA), which showed initial cam-post engagement at $100^{\circ}$ [18]. Both of these implants showed satisfactory long-term clinical results $[1,19]$. The Zimmer Persona TKA is characterized by side-specific implants, an increase in size selection having gender specific measures, anatomical asymmetric tibial plates, and a femur-shaped cutting jig (Figure 4).

The authors of the current study compared the PS box cutting jigs, divided in small, medium, and large, which are part of the surgical instrumentation of these knee systems. All measurements showed that all implants require some bone resection: few studies correlated quantity of bone resection to implant selection in case of revision $[7,8]$. Our results indicate that some cutting jigs tested might remove over twice as much bone from the intercondylar notch; this is especially true in small and medium sizes.

Other than saving bone stock in prevision of a possible implant revision, resection of intercondylar bone may create a potential stress riser in the distal femur, predisposing to intercondylar fracture. In this study, we are not attempting to point out a better design at the expense of another, but simply to identify a less invasive surgical instrumentation if a PS solution is needed. The cam and peg mechanisms for these designs also have many differences with regards to size, position, and articulating geometries.

Very few studies compared bone loss between different types of PS implant devices. In 2000, Haas et al. [20] showed significant differences in bone loss between different PS designs. Unfortunately, none of them were modern, patella 'friendly' implants. Recently, Wragg et al. [2] compared the amount of sawbone excised between PS and cruciate retaining designs, showing that significantly more bone is excised using PS devices.

This study had several limitations. First, our measurements were performed in an in vitro environment: we compared the tridimensional box area required for the PS housing using different PS box cutting jigs and not the real quantity of bone removed during an in vivo box osteotomy. This area does not necessarily correspond with actual intraoperative bone resection. The amount of bone removable in an in vivo situation is variable and also dependent upon femoral condyles conformation and the level of distal femoral resection. Many intramedullary femoral resection guides provide a standard $10-\mathrm{mm}$ distal femur osteotomy. Secondarily, the clinical value of removing more or less bone during primary TKA has to be fully established. Our hypothesis, unfortunately not fully provable by this study, is that resection of a larger segment of intercondylar notch might have an influence on in vivo knee kinematics, polyethylene wear, stability of the implant, and overall survivorship. On the other side, we agree with the previous studies reporting on the need of more invasive revision fixation devices (cones, methaphyseal sleeves, and/ or stems) in the case of extensive bone loss [7].

\section{Conclusions}

This study shows that once a PS total knee arthroplasty design is chosen, some extra bone resection must to be 
taken in account. Differences exist in the tridimensional PS mechanism housing area of different PS TKA designs. Surgeons still have various options when they select a TKA design: CR or ultra-congruent implants, which do not need accessory bone resection for the PS mechanism housing, have shown excellent long-term clinical results.

\section{Competing interests}

The authors declare that they have no competing interests.

\section{Authors' contributions}

PFI conceived the study, participated in its design and coordination, and originally drafted the manuscript. LL participated in the design of the study and performed the statistical analysis. SC performed the instrumentation measurements. AG participated in the design of the study and was responsible for the images section. MM selected the references and reviewed the final manuscript. All authors read and approved the final manuscript.

\section{Author details}

${ }^{1}$ Centro Eccellenza Sostituzioni Articolari Toscana (CESAT), Clinica Ortopedica Universita' di Firenze, Florence 50134, Italy. 'Fondazione Onlus "In Cammino", Piazza Lavagnini 1, Fucecchio, Florence 50054, Italy. ${ }^{3}$ Breyer Center for Overseas Study, Stanford University in Florence, Florence 50100, Italy. ${ }^{4}$ Clinica Ortopedica Universita' di Catania, Catania 95121, Italy. ${ }^{5}$ L.U.de.S. Libera Universita' degli Studi di Scienze Umane e Tecnologiche, Lugano 6900, Switzerland.

Received: 9 January 2014 Accepted: 16 June 2014

Published: 19 July 2014

\section{References}

1. Indelli PF, Aglietti P, Buzzi R, Baldini A: The Insall-Burstein II prosthesis: a 5- to 9-year follow-up study in osteoarthritic knees. J Arthroplasty 2002, 17(5):544-549.

2. Wragg R, Khan RJZ, Damasena ITW: A comparison of bone loss at total knee replacement: posterior stabilized versus cruciate retaining. J Orthopaedics 2012, 9(1):e1.

3. Becher C, Heyse TJ, Kron N, Ostermeier S, Hurschler C, Schofer MD, Fuchs-Winkelmann S, Tibesku CO: Posterior stabilized TKA reduce patellofemoral contact pressure compared with cruciate retaining TKA in vitro. Knee Surg Sports Traumatol Arthrosc 2009, 17(10):1159-1165.

4. Puloski SK, McCalden RW, MacDonald SJ, Rorabeck CH, Bourne RB: Tibial post wear in posterior stabilized total knee arthroplasty. An unrecognized source of polyethylene debris. J Bone Joint Surg Am 2001, 83-A(3):390-397.

5. Indelli PF, Marcucci M, Pipino G, Charlton S, Carulli C, Innocenti M: The effects of femoral component design on the patello-femoral joint in a PS total knee arthroplasty. Arch Orthop Trauma Surg 2013, 134:59-64 [Epub ahead of print].

6. Rodriguez-Merchan EC: Instability following total knee arthroplasty. HSS J 2011, 7(3):273-278

7. Bono JV, Scott RD (Eds): Revision total knee arthroplasty. New York: Springer; 2005.

8. Huten D: Femorotibial bone loss during revision total knee arthroplasty. Orthop Traumatol Surg Res 2013, 99S:S22-S33.

9. Pagnano MW, Cushner FD, Scott WN: The role of the posterior cruciate ligament in total knee arthroplasty. J Am Acad Orthop Surg 1998, 6:176-187.

10. Joglekar S, Gioe TJ, Yoon P, Schwartz MH: Gait analysis comparison of cruciate retaining and substituting TKA following PCL sacrifice. Knee 2012, 19(4):279-285.

11. Zimlitski M, Bhokray KK, Rajan BM, Nachkebia L, Loria G: Total knee arthroplasty with NexGen legacy posterior stabilized. Georgian Med News 2011, 199:14-20.

12. Li G, Zayontz S, Most E, Otterberg E, Sabbag K, Rubash HE: Cruciate-retaining and cruciate-substituting total knee arthroplasty: an in vitro comparison of the kinematics under muscle loads. J Arthroplasty 2001, 16(8 Suppl 1):150-156.
13. Lee SM, Seong SC, Lee S, Choi WC, Lee MC: Outcomes of the different types of total knee arthroplasty with the identical femoral geometry. Knee Surg Relat Res 2012, 24(4):214-220.

14. Hanusch B, Lou TN, Warriner G, Hui A, Gregg P: Functional outcome of PFC Sigma fixed and rotating-platform total knee arthroplasty. A prospective randomized controlled trial. Int Orthop 2010, 34:349-354.

15. Ranawat AS, Ranawat CS, Slamin JE, Dennis DA: Patellar crepitation in the P.F.C. sigma total knee system. Orthopedics 2006, 29(9 Suppl):68-70.

16. Stiehl JB, Dennis DA, Komistek RD, Keblish PA: In vivo kinematic comparison of posterior cruciate ligament retention or sacrifice with a mobile bearing total knee arthroplasty. Am J Knee Surg 2000, 13(1):13-18

17. Kievit AJ, Schafroth MU, Blankevoort L, Sierevelt IN, van Dijk CN, van Geenen RC: Early experience with the Vanguard complete total knee system: 2-7 years of follow-up and risk factors for revision. J Arthroplasty 2013, 29:348-354 [Epub ahead of print].

18. Moynihan A, Varadarajan K, Hanson GR, Park S, Nha KW, Suggs JF, Johnson T, Li G: In vivo knee kinematics during high flexion after a posterior-substituting total knee arthroplasty. Int Orthop 2010, 34(4):497-503.

19. Seon JK, Park JK, Shin YJ, Seo HY, Lee KB, Song EK: Comparisons of kinematics and range of motion in high-flexion total knee arthroplasty: cruciate retaining vs. substituting designs. Knee Surg Sports Traumatol Arthrosc 2011, 19(12):2016-2022.

20. Haas SB, Nelson CL, Laskin RS: Posterior stabilized knee arthroplasty: an assessment of bone resection. Knee 2000, 7:25-29.

doi:10.1186/s13018-014-0054-y

Cite this article as: Indelli et al:: Primary posterior stabilized total knee arthroplasty: analysis of different instrumentation. Journal of Orthopaedic Surgery and Research 2014 9:54.

\section{Submit your next manuscript to BioMed Central and take full advantage of:}

- Convenient online submission

- Thorough peer review

- No space constraints or color figure charges

- Immediate publication on acceptance

- Inclusion in PubMed, CAS, Scopus and Google Scholar

- Research which is freely available for redistribution 